\title{
Modal skepticism and counterfactual knowledge
}

\author{
Juhani Yli-Vakkuri
}

(C) Springer Science+Business Media B.V. 2012

\begin{abstract}
Timothy Williamson has recently proposed to undermine modal skepticism by appealing to the reducibility of modal to counterfactual logic (Reducibility). Central to Williamson's strategy is the claim that use of the same nondeductive mode of inference (counterfactual development, or $C D$ ) whereby we typically arrive at knowledge of counterfactuals suffices for arriving at knowledge of metaphysical necessity via Reducibility. Granting Reducibility, I ask whether the use of CD plays any essential role in a Reducibility-based reply to two kinds of modal skepticism. I argue that its use is entirely dispensable, and that Reducibility makes available replies to modal skeptics which show certain propositions to be metaphysically necessary by deductive arguments from premises the modal skeptic accepts can be known.
\end{abstract}

Keywords Metaphysical modality · Modal skepticism · Counterfactuals · Modal epistemology · Philosophical methodology

\section{Introduction}

Williamson (2007) has recently proposed to undermine modal skepticism by appealing to the reducibility of modal to counterfactual logic (Reducibility). Central to Williamson's strategy is the claim that use of the same non-deductive mode of inference (counterfactual development, or $C D$ ) whereby we typically arrive at knowledge of counterfactuals suffices for arriving at knowledge of metaphysical necessity via Reducibility. Granting Reducibility, I ask whether the use of CD plays any essential role in a Reducibility-based reply to two kinds of modal skepticism.

\footnotetext{
J. Yli-Vakkuri $(\bowtie)$

Wolfson College, Oxford University, Oxford OX2 6UD, UK

e-mail: juhani.yli-vakkuri@philosophy.ox.ac.uk
} 
I argue that its use is entirely dispensable, and that Reducibility makes available replies to modal skeptics which show certain propositions to be metaphysically necessary by deductive arguments from premises the modal skeptic accepts can be known.

I distinguish two sorts of modal skeptic-the Quinean and the Humean-and give a CD-free, Reducibility-based reply to each. The Quinean denies that one can ever know anything to be metaphysically necessary, but is not a skeptic about logical, mathematical, counterfactual, or other not-specifically-metaphysical subject matter. My reply to the Quinean uses Reducibility to argue, on general grounds, that any logical truth and any provable mathematical truth is metaphysically necessary. One could also use CD in replying to the Quinean, but the general deductive arguments are more decisive. The Humean focuses her skepticism more narrowly: she grants that one can have knowledge of the metaphysical necessity of logical and provable mathematical truths, but denies that one can have knowledge of the necessity of any proposition that is properly metaphysically necessary (i.e., metaphysically necessary but not necessary in any stricter sense). Like the Quinean, the Humean is not a skeptic about counterfactuals or other non-metaphysical subject matter. My reply to the Humean uses Reducibility to show that, as a matter of logic, both first-order identities and property identities are necessary if true (and necessarily false if false), and many of the standard examples of properly metaphysical necessities are logical consequences of true first- or second-order identities, which the Humean allows we can know. In the case of the Humean, a reply which uses CD does not even appear to be available to the modal epistemologist: the style of reply suggested by Williamson is not in accord with our ordinary way of evaluating counterfactuals. If he thinks CD can be successfully deployed against the Humean, then Williamson owes us an explanation of why the departure from ordinary counterfactual evaluation he recommends is legitimate. I argue, however, that all of the explanations on offer either make CD dispensable to Williamson's reply to the Humean or are inadequate for some other reason.

\section{Counterfactual knowledge versus modal skepticism}

Modal epistemology is concerned with our knowledge of metaphysical possibility and necessity. ${ }^{1}$ One task of modal epistemology is to construct an account of modal knowledge with the resources to reply to a kind of skeptic who claims that we know much less than we think we do about what is (metaphysically) necessary and possible. Williamson has recently taken on this task in Chap. 5 of The Philosophy of Philosophy (Williamson 2007, henceforth PoP).

One can distinguish any number of varieties of modal skepticism, but I will restrict my attention to two kinds that target knowledge of necessity rather than of possibility. I will be setting aside what might be called Arnauldian modal

\footnotetext{
${ }^{1}$ All reference hereafter to modality is to metaphysical modality unless context indicates otherwise.
} 
skepticism $^{2}$ : the claim that, for all false propositions $p$, we never know that $\diamond p{ }^{3}$ I leave it an open question whether Williamson has an adequate reply to the Arnauldian, and whether his reply does any better than conceivability-based replies such as Yablo's (1993).

The first kind of skepticism I consider is Quinean modal skepticism:

QMS For all propositions $p$, we never know that $\square p$.

The Quinean modal skeptic is not a skeptic about counterfactuals or other notspecifically-metaphysical subject matter: she allows that we can know all the sorts of things we take ourselves to know outside of the metaphysics seminar, but never that something is necessarily so.

Another variety of modal skepticism concerns knowledge of properly metaphysical necessities. A truth is properly metaphysically necessary (a $P M N)$ iff it is metaphysically necessary but not necessary in any stricter sense. (A necessity $\square_{1}$ is stricter than $\square_{2}$ iff: $\square_{1} p \rightarrow \square_{2} p$, for all $p$, but the converse does not hold. ${ }^{4,5}$ ) Logical, mathematical, and conceptual or analytic necessity are necessities stricter than metaphysical necessity. Paradigm cases of PMNs are a posteriori necessary truths such as: that Hesperus is Phosphorus, that gold has atomic number 79, that water contains oxygen, etc. ${ }^{6}$ The second kind of skeptic-the Humean modal skeptic-acknowledges that we can know many things to be metaphysically necessary, but maintains that:

HMS If $p$ is a PMN, then we never know that $\square p$.

Unlike the Quinean's position, the Humean's is nowadays widely held, and even more widely treated as a serious threat to modal metaphysics. One could be a Humean for any number of reasons, but the reason that strikes me as most plausible is this: alleged PMNs are all a posteriori; the negation of any a posteriori proposition is conceivable, and inferences from conceivability to possibility appear to be what underwrite our knowledge of metaphysical possibility: "if there is a seriously alternative basis for possibility theses, then philosophers have not discovered it yet" (Yablo 1993, p. 2). If our only method of assessing propositions for metaphysical

\footnotetext{
${ }^{2}$ I make no claims about the historical accuracy of the labels for the kinds of modal scepticism defined here.

${ }^{3}$ See Yablo (1993) for discussion.

${ }^{4}$ The case of alleged contingent logical truths might seem to preclude the classification of logical and analytic necessity as stricter than metaphysical necessity, contrary to my intention. See $\$ 5$ below on contingent logical truths. For someone who doubts that there is any necessity stricter than metaphysical necessity, we can define properly metaphysical necessities some other way-e.g., as necessary a posteriori truths (thanks to Margot Strohminger for this suggestion).

${ }^{5}$ Speaking of logical necessity as a property of propositions will appear problematic for the traditional Millian who maintains that 'Marilyn Monroe = Marilyn Monroe' and 'Marilyn Monroe = Norma Jean Baker' express the same proposition (the first sentence is logically true, the second not; what is the logical status of the proposition expressed?). Although Millians have tended to be sympathetic towards the view that propositions have sentence-like structure, they have not, until recently, made use of all of the distinctions which the conception of propositions as structured allows them to make: see Putnam (1954) and Fine (2007).

${ }^{6}$ See Kripke (1971/1977, 1980) and Putnam (1973, 1975).
} 
possibility classifies the negations of alleged PMNs as metaphysically possible, how could we ever know a PMN, if there are any such things, to be necessary? ${ }^{7}$

The Humean's challenge to the modal epistemologist, then, is to explain how we could come to know any PMN to be necessary. The Quinean's challenge is to explain how we could know any proposition to be necessary.

I think, and Williamson presumably agrees, that a reply to a modal skeptic of any kind should meet two desiderata:

\section{Conservatism}

It should turn out that we can at least have knowledge of necessity for a representative range of paradigm cases of propositions that we take ourselves to know to be necessary-within the class of propositions to which the skeptic applies her skepticism. That is, if the skeptic claims that we cannot know any proposition of type A to be necessary, it should be a consequence of our reply that we can know the necessity of a representative range of paradigm cases of A-type propositions we take ourselves to know to be necessary.

\section{Anti-exceptionalism}

"[A] plausible non-skeptical epistemology of metaphysical modality should subsume our capacity to discriminate metaphysical possibilities from metaphysical impossibilities under more general cognitive capacities used in ordinary life" (PoP, p. 136).

The motivation for Conservatism is obvious: it would be a small comfort if, say, we succeeded in refuting the skeptic by showing that we have knowledge of the necessity of just one proposition in the targeted class but were unable to show that we can know anything else to be necessary. As for Anti-exceptionalism, I agree with Williamson that "the postulation by philosophers of a special cognitive capacity exclusive to philosophical or quasi-philosophical thinking looks like a scam" (ibid.).

My aim will be to show how Reducibility can be used to supply an account that satisfies both desiderata. However, I argue, pace Williamson, that such an account

\footnotetext{
${ }^{7}$ Many papers in modal epistemology (see Gendler and Hawthorne 2002 for a sample and references) attempt to rebut this kind of motivation for modal skepticism. Yablo (1993) is a classic example. Hill (2006) is a more recent one. Just like the Humean, Hill worries that "Coherent conceiving is a reliable test for conceptual possibility, but not for metaphysical possibility" (p. 229). This observation motivates his search for an account of modal knowledge based on something other than conceiving alone. Something similar can be said of Williamson himself, who writes that "it is imaginable but not possible that water does not contain oxygen, except in artificial senses of 'imaginable' that come apart from possibility in other ways" $(P o P$, p. 163). The prominence of the Humean's position in the literature is undeniable, even though it is difficult to find examples of philosophers who explicitly endorse Humean modal skepticism in print. Nevertheless, conversations with philosophers have led me to believe the position to be widespread (a prominent example: Stephen Stich, in conversation, unhesitatingly assented to the Humean position when I described it to him). I suspect that the paucity of textual evidence of widespread commitment to the Humean position is due to the fact that philosophers who subscribe to it generally do not write about modality. What evidence there is typically comes in the form of an explanation of one's refusal to engage with the topic of the necessary a posteriori, on the grounds that, allegedly, conceivability is the only known way of assessing the modal status of propositions (Segal 2000, p. 15f, is a good example of this). Others (e.g., Fitch 1976; Swinburne 1991) have denied that there are any necessary a posteriori truths. Because one cannot know what is not true, these philosophers are also Humeans.
} 
should not be framed in terms of the non-deductive mode of inference (counterfactual development) whereby we typically arrive at knowledge of counterfactuals.

Williamson's account of modal knowledge begins with the observation that the logic of metaphysical modality is reducible to counterfactual logic via the equivalence

$$
\begin{aligned}
& \text { Reducibility } \\
& \square p \equiv \neg p \square \longrightarrow \perp,
\end{aligned}
$$

from which $\diamond p \equiv \neg(p \square \longrightarrow \perp)$ follows by the duality of $\diamond$ and $\square$. Exploiting these equivalences, we may, as Williamson notes, use the same cognitive capacities by which we evaluate counterfactuals to evaluate modal claims: "the ordinary cognitive capacity to handle counterfactual conditionals carries with it the cognitive capacity to handle metaphysical modality" (PoP, p. 136). The capacity for counterfactual thinking is a thoroughly ordinary cognitive capacity, so Antiexceptionalism is respected-provided that Williamson can show that ordinary capacity is capable of producing knowledge of counterfactuals that are logically equivalent to modal claims.

The qualification just made bears some emphasis. Reducibility alone does not guarantee the satisfaction of Anti-exceptionalism. That the ability to evaluate counterfactuals itself is thoroughly ordinary does not rule out the possibility that the evaluation of some counterfactuals requires other, less than ordinary capacities due to the material in their antecedents and consequents. For example, if it turned out that the evaluation of any counterfactual with a contradiction as a consequent required a special faculty of rational intuition not put to use outside of philosophy, Anti-exceptionalism would be violated. ${ }^{8}$ It is therefore incumbent upon Williamson to show that we can come to know not only humdrum counterfactuals but specifically those counterfactuals which are equivalent to necessity claims-or at least a representative range of them-by using only ordinary cognitive capacities. I think this challenge can be met.

Meeting this challenge, however, does not require showing that "knowing certain counterfactuals is the way, or even our usual way, of knowing modal facts" (Jenkins 2008, p. 695). Jenkins correctly points out that Reducibility entails no such thing, and that "[a]t most, [Reducibility] might be taken to suggest that knowing the relevant counterfactuals is $a$ way of coming to know modal facts" (ibid.). While Jenkins may be right that "epistemologists of modality are (rightly) more centrally interested in how we do know about modality than in the question of how we might know about modality" (pp. 695-696), I take it that the anti-skeptical project in which Williamson and I are engaged is well enough served by the finding that we

\footnotetext{
${ }^{8}$ Malmgren (forthcoming: §3) raises a related worry: that the evaluation of those counterfactuals that, according to Williamson, capture the verdicts issuing from philosophical thought experiments such as Gettier cases might require "rational intuition". Though this would be bad news for Williamson's overall metaphilosophical project, it would have no effect on the project of fending off skepticism about modality. I am not concerned with responding to the sort of modal skeptic who (only) thinks we never have knowledge of the necessity of specifically philosophical claims. However, if the reply I develop in $\S 4$ is correct, then such a skeptic must be wrong-after all, very many interesting philosophical claims have the form of identity claims: that knowledge is justified true belief; that to have a disposition to $R$ when $S$ is to be such that if one were $R$-ed, then one would $S$; and so on.
} 
have an ordinary cognitive capacity which enables us to acquire knowledge of modality in paradigmatic cases in which we take ourselves to have modal knowledge. There is, of course, a further skeptical worry that can be introduced even if Williamson's project succeeds: perhaps, even though Williamson's approach shows that we could have modal knowledge in the paradigmatic cases, we actually arrived at our modal beliefs by some non-knowledge-conducive method which just happens to produce largely the same judgments as counterfactual reasoning plus Reducibility, and we therefore have no modal knowledge. However, such a skeptical scenario does not seem particularly worrying if Williamson's project succeeds. After all, once we are in possession of the counterfactual method for producing modal knowledge, we can use it to acquire knowledge of the various modal facts which we previously only believed.

As a first step in outlining a counterfactual method for producing modal knowledge, Williamson offers an account of how we evaluate counterfactuals in general. He describes the assertibility conditions of counterfactuals as follows.

[W] e assert $\mathbf{A} \square \mathbf{B}$ when our counterfactual development of the supposition $\mathbf{A}$ robustly yields $\mathbf{B}$; we deny $\mathbf{A} \square \longrightarrow \mathbf{B}$ when our counterfactual development of $\mathbf{A}$ does not robustly yield $\mathbf{B}$ (and we do not attribute the failure to a defect in our search) (PoP, p. 163).

These conditions are then applied to modal claims via Reducibility:

[W] e assert $\square \mathbf{A}$ when our counterfactual development of the supposition $\neg \mathbf{A}$ robustly yields a contradiction; we deny $\square \mathbf{A}$ when our counterfactual development of $\neg \mathbf{A}$ does not robustly yield a contradiction (and we do not attribute the failure to a defect in our search) (ibid.).

The underlying cognitive capacity is one for a non-deductive form of inference Williamson calls the counterfactual development of a supposition-I will simply call it counterfactual development $(C D)$. Williamson describes $\mathrm{CD}$ as follows:

We can... schematize a typical overall process of evaluating a counterfactual conditional thus: one supposes the antecedent and develops the supposition, adding further judgments within the supposition by reasoning, offline predictive mechanisms, and other offline judgments. The imagining may but need not be perceptual imagining. All of one's background knowledge and beliefs are available from within the scope of the counterfactual supposition as a description of one's actual circumstances (if we know $\mathbf{B}$, we can infer A $\square \longrightarrow$ B for any A...). Some but not all of one's background knowledge and beliefs are also available within the scope of the supposition as a description of the counterfactual circumstances, according to complex criteria (the problem of cotenability). To a first approximation: one asserts the counterfactual if and only if the development eventually leads one to add the consequent (PoP, p. 153).

Let us assume Reducibility, and assume that Williamson's description of the way we typically evaluate counterfactuals is correct. Given these assumptions, what role might $C D$ play in a Reducibility-based reply to a modal skeptic? A natural 
suggestion here would be that we can reply to a skeptic who denies we can know $\square p$ by exhibiting a CD of $\neg p$ into $\perp$, on the basis of which we conclude that $\neg p \square \longrightarrow \perp$ and, by Reducibility, $\square p$. But, in fact, the reply need not ever take that form, and in some cases it is unclear whether it can take that form. There are adequate replies to modal skeptics of both the Quinean and Humean variety which never exhibit even one $\mathrm{CD}$, but instead show that we can know the necessity of whole classes of propositions on the basis of general logical and epistemological considerations. Furthermore, it is doubtful that a reply that makes use of CDs is even available in the case of the Humean. In the next two sections I show how to use Reducibility to reply to the Quinean and Humean without engaging in CD.

\section{Replying to the Quinean}

I begin with the Quinean, who professes not to understand modal talk when the modality in question is metaphysical. Thanks to Reducibility, we can explain the intended senses of the modal words to her in terms she does understand: the counterfactual conditional and truth-functional connectives. We can say to her that $\square p$ simply means $\neg p \square \longrightarrow \perp$. Reducibility can be used to uniquely single out the metaphysical sense of 'necessary'.?

The next step is to show that we can use Reducibility to arrive at knowledge of necessity. This will be straightforward in the case of any mathematical truth for which there is a proof, and for any logical truth. Neither case requires us to engage in CD.

Let us consider mathematical truths first. It is a logical platitude that any claim that has a proof has a proof by reductio. If $p$ is a provable mathematical truth, it is easy to show $p$ to be necessary by proving $p$ by reductio. A proof of $p$ by reductio is just a special case of a CD of the supposition that $\neg p$ into a contradiction. In a reductio, one makes the counterfactual supposition that $\neg p$. One then imports into the scope of the supposition some further facts $q_{1}, \ldots, q_{n}$, and one gives a proof of $\perp$ from $\left\{\neg p, q_{1}, \ldots, q_{n}\right\}$. Clearly, if a counterfactual supposition of $\neg p$ together with facts legitimately imported into its scope deductively yields $\perp$, then $\neg p \square \longmapsto \perp$, and, by Reducibility, $\square p$.

The argument just given is a deductive argument for the conclusion that every provable mathematical truth is necessary. Nowhere does it make use of CD-it would not be deductive if it did-though it of course makes use of an assumption about $\mathrm{CD}$ : that a proof by reductio is a $\mathrm{CD}$ of the negation of what is to be proved into $\perp$. But this is hardly controversial if we accept Williamson's account of the assertibility conditions of counterfactuals.

The argument in the case of logical truths is even more general: one can appeal to general logical principles to prove via Reducibility, in one fell swoop, that every logical truth is necessary. For let $p$ be a logical truth. Then $\neg p \rightarrow \perp$ is a logical

\footnotetext{
${ }^{9}$ Up to logical equivalence, at least. One could argue that, because they differ in semantic structure, the two sides of the equivalence in Reducibility are not strictly synonymous (see PoP, p. 160).
} 
truth. If a material conditional is logically true, then the corresponding counterfactual is too, so $\neg p \square \longrightarrow \perp$ is a logical truth. ${ }^{10}$ By Reducibility, $\square p$ is a logical truth as well. ${ }^{11}$ Whenever $p$ is a logical truth, so is $\square p$. (This, of course, is a proof of the validity of the "Necessitation" rule of normal modal logics—viz.: if $\vdash p$ then $\vdash$ $\square p.)^{12}$

There is nothing for the Quinean to object to here. She is a skeptic specifically about metaphysical necessity, not about counterfactuals, or about logical or mathematical truth. Because she understands counterfactuals and truth-functional connectives, she understands $\square$ thanks to Reducibility. Because she understands counterfactuals, she knows that any proof by reductio can be converted into a CD that yields a contradiction, and she knows by Reducibility that every mathematical truth for which there is a proof is necessary. Because she understands counterfactuals, she knows that $p \square \longrightarrow q$ is a logical truth whenever $p \rightarrow q$ is, and this is all that is required for a proof of the necessity of all logical truths via Reducibility. Conservatism is respected: we can know that all logical truths are necessary, and that at least any provable mathematical truth is. And at no point in my reply did I engage in CD.

\section{Replying to the Humean}

Few philosophers nowadays accept the extreme, Quinean version of modal skepticism, however. The typical contemporary modal skeptic is of the Humean variety. Let us suppose that the Humean recognizes the adequacy of $\S 3$ 's reply to the Quinean: she accepts the characterization of metaphysical necessity in terms of counterfactuals, and accepts that we know many things to be necessary-among them logical and provable mathematical truths. But she denies that we can know any properly metaphysical necessities (PMNs) - propositions which are metaphysically necessary but not necessary in any stricter sense- to be necessary. Her reason for denying this is that the negations of PMNs are conceivable.

One might think that simply by providing an alternative to the conceivability test commonly attributed to Hume (judge that $\diamond_{p}$ if $p$ is conceivable), Reducibility constitutes an adequate reply to the Humean: Never mind if $\neg p$ is conceivable

\footnotetext{
${ }^{10}$ The argument relies on the quite innocent rule of Deduction, viz.: if $\vdash p \rightarrow q$ then $\vdash p \square \mapsto q$, which is common to both Lewis's and Stalnaker's counterfactual logics. In Lewis's system, a more general version of this rule is called "Deduction within Conditionals" (1973, p. 132). In Stalnaker's system Deduction follows from axiom schema (a1) via Necessitation and the definition of $\square$ (Stalnaker 1968/1981, pp. 105-106).

${ }^{11}$ This proof (and the rule of Deduction) is invalid if there are contingent logical truths. I address this concern in $\S 5$.

${ }^{12}$ It is worth noting that paradigm cases of analytic truths can also be shown to be necessary by an extension of this argument. According to one traditional view, all analytic truths can be obtained from logical truths by substituting synonyms for synonyms. Whether or not this is true, at least the paradigm cases of analytic truths have this feature: e.g., 'All bachelors are unmarried' is presumably analytic because 'bachelor' is synonymous with 'unmarried man'. To show that it is necessary that all bachelors are unmarried, then, we simply show that 'All unmarried men are unmarried', a logical truth, is necessary, and then replace 'unmarried men' with 'bachelors'.
} 
whenever $p$ is a PMN; if you know that $\neg p \square \longrightarrow \perp$, then you know that $\square p$ ! But this is not so: nothing that has been said so far gives any indication of how one might come to know that $\neg p \square \perp$ when $p$ is a PMN. The Humean can (justly, I argue in §5) complain that whenever $\neg p$ is conceivable, we do not know how to counterfactually develop $\neg p$ into a contradiction. CD appears to be of no help here.

It is, then, a good thing that there is a Reducibility-based reply to the Humean which makes no use of CD. This reply simply points out that, for a broad class of PMNs $p, \square p$ is a logical consequence of propositions the Humean grants that we know.

Let us begin with identity claims. That Hesperus $=$ Phosphorus $(\mathrm{H}=\mathrm{P})$ is a PMN. Because it is a logical truth that $\mathrm{H}=\mathrm{H}$, by the argument of $\S 3$ it is also a logical truth that $\mathrm{H} \neq \mathrm{H} \square \longrightarrow \perp \mathrm{H} \neq \mathrm{P} \square \longrightarrow \perp$ follows by Leibniz's Law from $\mathrm{H} \neq \mathrm{H} \square \longrightarrow \perp$ and $\mathrm{H}=\mathrm{P}$, which the Humean will agree that we know. By Reducibility, we conclude that $\square \mathrm{H}=\mathrm{P}$, and this, being deduced from a known premise, is also known.

The necessity of the distinctness of Hesperus and Jupiter ( $\mathrm{J})$ is also easy to deduce from a known premise: that $\mathrm{H} \neq \mathrm{J}$. By the argument of the previous paragraph,

$$
(*) \mathrm{H}=\mathbf{J} \square \longrightarrow(\mathrm{H} \neq \mathbf{J} \square \longrightarrow \perp)
$$

is a logical truth. The inference from $(*)$ and $\mathrm{H} \neq \mathrm{J}$ to $\mathrm{H}=\mathrm{J} \square \longrightarrow \perp$ is obviously valid (it is a propositional counterfactual logic validity: the argument is of the form $\neg p, p \square \longrightarrow(\neg p \square \longrightarrow \perp) / \therefore p \square \longrightarrow \perp)$. By Reducibility, $\mathrm{H}=\mathrm{J} \square \longrightarrow \perp$ entails $\square \mathrm{H} \neq \mathrm{J}$.

There is nothing for the Humean to object to here. She understands counterfactuals, so she is capable of recognizing the validity of simple valid arguments involving counterfactuals. The arguments for the necessity of the identity of Hesperus and Phosphorus and of the distinctness of Hesperus and Jupiter are simple valid arguments involving counterfactuals.

We can also use deductive arguments to show that a wide variety of paradigm examples of PMNs which are not identity or distinctness claims are necessary, because they are logical consequences of true identity or distinctness claims. For example, much ink has been spilled over whether 'Water is $\mathrm{H}_{2} \mathrm{O}$ ' has the logical form of an identity (see Soames 2002, Chaps. 10-11 and citations). Let us suppose that it does not, but that it has, as one hypothesis holds (see Parsons 1979), the logical form:

(W1) $\forall x\left(\boldsymbol{w a t e r}(x) \leftrightarrow \boldsymbol{H}_{2} \boldsymbol{O}(x)\right)$.

(According to Parsons, a mass term like 'water' is a predicate that is true of quantities or samples of stuff.) If so, then the following seems to be something we know.

(W2) $\lambda x . w a t e r(x)=\lambda x . H_{2} \boldsymbol{O}(x)$.

Since $\forall x(\boldsymbol{w a t e r}(x) \leftrightarrow \boldsymbol{w a t e r}(x))$ is a logical truth, so is

$$
\text { (W3) } \neg \forall x(\boldsymbol{w a t e r}(x) \leftrightarrow \boldsymbol{w a t e r}(x)) \square \longrightarrow \perp,
$$


and

$$
\text { (W4) } \neg \forall x\left(\boldsymbol{w a t e r}(x) \leftrightarrow \boldsymbol{H}_{2} \boldsymbol{O}(x)\right) \square \longrightarrow \perp
$$

follows from (W2) and (W3) by the second-order variant of Leibniz's law and lambda conversion. By Reducibility, (W4) entails the necessity of (W1). We can deal similarly with 'Whatever has a temperature of $30^{\circ} \mathrm{C}$ has a mean molecular kinetic energy of $30^{\circ} \mathrm{C}$ ', and many other standard examples. We can, of course, also show the necessity of the property identity claims themselves via a second-order variant of the argument for the necessity that $\mathrm{H}=\mathrm{P}$.

The Humean must grant that we do, at least in some cases, know property identities; she is only a skeptic concerning the necessity of PMNs, not a skeptic about identity. There are many property identities we know empirically: that the property of being (a quantity of) gold is the property of being (a quantity of) element \#79, that the property of having temperature-measured-in-Celsius of 30 is the property of having mean molecular kinetic energy-measured-in-Celsius of 30, and so on. For a modal skeptic to suggest that gold and element \#79, temperatures of $30^{\circ} \mathrm{C}$ and mean molecular kinetic energies of $30^{\circ} \mathrm{C}$, etc., might be always compresent despite being distinct would be no more motivated than to suggest that Hesperus and Phosphorus might be spatiotemporally coincident despite being distinct. Some reason must be given for why we do not know these identities, which we take ourselves to know outside of philosophical contexts.

Of course, the Humean must allow that we know even more property distinctness claims (whenever not every $P$ is a $Q, \lambda x . P x \neq \lambda x . Q x$ ), whose necessity deductively follows by the second-order version of the proof of the necessity of distinctness, and a vast number of such truths are PMNs. The Humean must allow, then, that we know very many PMNs, including a representative range of the paradigm cases, to be necessary.

Like my reply to the Quinean, my reply to the Humean never makes use of CD. It shows that very many PMNs can be known to be necessary because their necessity can be deduced from known premises. Unlike my reply to the Quinean, my reply to the Humean does not even mention $\mathrm{CD}$, and it is unclear what good mentioning or using CD could do in this context, as we shall see in the next section.

\section{Williamson's alternative}

Williamson pursues a different tack than I have in $\S \S 3-4$, making use of CD where I have used deductive arguments. In this section I contrast his approach with mine.

I begin with mathematical truths. One can indeed use $\mathrm{CD}$ to show that a provable mathematical truth is necessary, because, again, a proof by reductio just is a CD of a supposition into a contradiction. But in order to do this one must know how to prove the truth whose necessity is in question. There are a vast number of provable mathematical truths we do not know how to prove, so presumably a reply to the Quinean which made use of $\mathrm{CD}$ would have to make an inductive case for the necessity of provable mathematical truths: one exhibits a sufficient number of CDs of the negations of 
mathematical truths one knows how to prove into contradictions, and then one projects the hypothesis that all provable mathematical truths are necessary.

Whether or not such an induction is warranted, there is something deeply unsatisfying about this way of replying to the Quinean. It misses the reason why we succeed in showing any provable mathematical truth to be necessary by CD: that whatever is provable is provable by reductio, and that a proof by reductio is ipso facto, thanks to Reducibility, warrant for the assertion of a necessity claim. To show this, one need not ever counterfactually develop the negation of a provable mathematical truth into a contradiction, nor does it help to do so (except perhaps pedagogically: one might hit upon the deductive argument by considering a variety of CDs of provable mathematical truths into contradictions). In replying to the Humean, the deductive argument for the necessity of all provable mathematical truths is by far preferable to the CD-based inductive argument for the same.

One could also use CD to argue that various logical truths are necessary, by proving them by reductio, and projecting the hypothesis that all provable logical truths are necessary. But in doing so one would miss an important logical fact: that whenever $p$ is a logical truth (provable or not), so is $\neg p \square \longrightarrow \perp$. Surely a deductive argument that uses this fact to show that all logical truths are necessary is preferable as a reply to the Quinean.

At this point, however, I must consider an objection that may be extrapolated from Williamson's discussion ( $P o P$, p. 144). My argument for the necessity of all logical truths is valid only if there are no contingent logical truths, for if $p$ is a contingent logical truth, then the logical truth of $\neg p \rightarrow \perp$ does not guarantee the truth, let alone logical truth, of $\neg p \square \longrightarrow \perp$. And if the logical truth of $\neg p \rightarrow \perp$ does not guarantee the truth of $\neg p \square \longrightarrow \perp$, then, not only is my argument for the necessity of all logical truths invalid, but even the CD-based method suggested above as an alternative to it will fail, and so will the CD-based method suggested above as an alternative to my argument for the necessity of all provable mathematical truths. Both methods presuppose that a proof by reductio of $p$ is a legitimate CD of $\neg p$ into $\perp$. But this will not be the case if $p$ is a contingent logical truth, or if one of the ancillary assumptions used in the derivation of the contradiction from $\neg p$ is. If there are contingent logical truths, then, if CDs are at all usable for arriving at knowledge of necessity, then CDs cannot be reconstructed as proofs by reductio.

I have no interest in defending the claim that every $\mathrm{CD}$ by which we could arrive at knowledge of necessity can be reconstructed as a deductive argument. And certainly Williamson is right that CDs generally cannot be so reconstructed. However, I think it would be a particularly bad consequence if the pre-theoretically obvious connection between counterfactuals and proofs by reductio-to wit, that if there is a proof by reductio of $p$, then $\neg p \square \longrightarrow \perp$ (see Lewis 1973, p. 24)-were severed. In countenancing contingent logical truths, one incurs great systemic costs, of which this is one. Other systemic costs include: that the so-called Deduction rule of counterfactual logics ${ }^{13}$ becomes invalid, and that the substitution of logical equivalents becomes invalid. These costs are good reasons to reject the claim that

\footnotetext{
13 See Lewis (1973, p. 132) and Stalnaker (1968/1981, pp. 105-106).
} 
there are contingent logical truths. I also note that the standard examples ( $p$ iff actually $p$ ', 'I am here now') are contentious for independent reasons. ${ }^{14}$

However, I prefer not to leave the adequacy of my reply to the Quinean to turn on the vexed question of whether there are contingent logical truths. The following is a simple modification of $\S 3$ 's argument for the necessity of logical truths which allows for the existence of contingent logical truths. Let us call logical truths which do not contain 'actually', 'I', or any of the other words that allegedly give rise to contingent logical truths, secure. If $p$ is secure, then so is $\neg p \rightarrow \perp$; and if $\neg p \rightarrow \perp$ is secure, then $\neg p \square \longrightarrow \perp$ is secure, and therefore so is $\square p$. Because any secure sentence is logically true, and therefore true, according to more permissive conceptions of logical truth, we can have a proof of the necessity of a large class of logical truths - the secure ones-regardless of whether there are any contingent logical truths. And my argument for the necessity of all provable mathematical truths would remain entirely unaffected, because the derivation of a contradiction from the premises of a reductio argument for a purely mathematical claim is always secure, as it is expressed in indexical-free language.

Let us finally turn to the PMNs. I argued that knowledge of the necessity of a wide range of paradigmatic PMNs could be obtained by deduction from known identity and distinctness facts. Can it also be obtained by Williamson's preferred method, CD? In CD, again:

one supposes the antecedent and develops the supposition, adding further judgments within the supposition by reasoning, offline predictive mechanisms, and other offline judgments ... Some but not all of one's background knowledge and beliefs are... available within the scope of the supposition as a description of the counterfactual circumstances, according to complex criteria (the problem of cotenability) ... [O]ne asserts the counterfactual if and only if the development eventually leads one to add the consequent (PoP, p. 153).

\section{That}

(G) Gold has atomic number 79

is a paradigmatic PMN. Can we counterfactually develop the supposition that $\neg(\mathrm{G})$ into a contradiction by adding further judgments cotenable with it? It would seem that we cannot: there is no proof of $(\mathrm{G})$, so there is no proof of $(\mathrm{G})$ by reductio. How, then, could we counterfactually develop $\neg(\mathrm{G})$ into a contradiction? Williamson's reply here is surprising. He suggests that we import the fact that (G) into the scope of the counterfactual supposition that $\neg(\mathrm{G})$ :

If we know enough about chemistry, our counterfactual development of the supposition that gold is not the element with atomic number 79 will generate a contradiction. The reason is not simply because we know that gold is the

\footnotetext{
14 There is good evidence that 'actually' does not always (so to speak) take its operand back to the world of the context; rather, the world with respect to which its operand is evaluated can be shifted by other operators. (See Cresswell 1990, Chap. 3. This observation appears to have been made first in Saarinen 1977, pp. 25-27, though using 'in fact' instead of 'actually'.) On 'I am here now', see Predelli (1998).
} 
element with atomic number 79, for we can and must try to vary some items of our knowledge under counterfactual suppositions. Rather, part of the general way we develop counterfactual suppositions is to keep such constitutive facts fixed (PoP, p. 164, emphasis ours).

[W]e need not judge that it is metaphysically necessary that gold is the element with atomic number 79 before invoking the proposition that gold is the element with atomic number 79 in the development of the counterfactual supposition. Rather, projecting constitutive matters such as atomic numbers into counterfactual suppositions is part of our general way of assessing counterfactuals. The judgment of metaphysical necessity originates as the output of a procedure of that kind; it is not an independently generated input (PoP, p. 170, second emphasis ours).

The reply is that $(\mathrm{G})$ is a constitutive fact, and that every constitutive fact is cotenable with every counterfactual supposition, so $(\mathrm{G})$ is cotenable with $\neg(\mathrm{G})$.

Let us set aside for the moment the question of what a constitutive fact is.

That some propositions are cotenable with their negations is a surprising claim. It is no "part of our general way of assessing counterfactuals" to import $p$ into the scope of the supposition that $\neg p$. It may be part of our way of assessing counterfactuals to generally import constitutive facts, whatever they may be, into counterfactual suppositions, but when the counterfactual supposition is the negation of the very constitutive fact to be imported, they are not cotenable by the standards by which we ordinarily judge cotenability. Even in cases in which we import facts inconsistent with the supposition into its scope for the purpose of a reductio, we do not import the very fact of which the supposition is a negation. The suggestion that showing a PMN to be necessary requires one to import $p$ into the scope of the supposition that $\neg p$ is extraordinary, and requires some justification.

Williamson's justification is rather terse: a constitutive fact may be imported into the scope of any supposition whatever. But what is a constitutive fact? Williamson does not answer this question, but here are four of the most obvious ways in which it might be answered.

First, it might be that constitutive facts are just those facts that we import into every counterfactual supposition-or, anyway, that we import into every counterfactual supposition when we are evaluating counterfactuals correctly. If so, perhaps one can discover a fact to be constitutive by developing a range of counterfactual suppositions and performing an inference to the best explanation: e.g., if one considers a wide enough range of counterfactual suppositions and one finds that they all lead one to add $(G)$, one might conclude that every counterfactual supposition yields $(G){ }^{15}$ The problem with this inductive generalization is that, unless one is already convinced of its truth, it will appear to have obvious counterexamples: after all, the counterfactual supposition that $\neg(\mathrm{G})$ is prima facie not cotenable with $(\mathrm{G}){ }^{16}$

\footnotetext{
15 This appears to be Hill's (2006) suggestion. See his discussion of "two tests" for the necessity of $p$ (pp. 230-231).

16 Hill (2006) does not consider the case where the antecedent is the negation of the proposition whose necessity we are trying to show.
} 
Second, it might be that constitutive facts are just necessary truths. By Reducibility, whenever $p$ is a necessary truth, $\neg p \square q$ for all $q$. This justifies the importation of a necessary truth into the scope of any counterfactual supposition. But if the justification for the importation of $(\mathrm{G})$ into the scope of the supposition that $\neg(G)$ is that $(G)$ is necessary, then that justification is the very piece of knowledge our CD of $\neg(\mathrm{G})$ into $(\mathrm{G})$ was supposed to produce: that $(\mathrm{G})$ is necessary. No need for CD, then.

The second reply could also be developed in a way which does not render CD superfluous, but which would be inadequate for other reasons: the idea is that to correctly evaluate $\neg(\mathrm{G}) \square \longrightarrow(\mathrm{G})$, we need not know that (G) is necessary; it suffices that we are appropriately sensitive to necessary truths in performing CDs, so that we import them into the scope of every counterfactual supposition. The problem with this proposal is that it will appear to violate Anti-exceptionalism unless accompanied by some reason to think that this sensitivity to necessary truths is a capacity we put to use outside of philosophy.

Third, it might be that constitutive facts are those which concern the constitutive or essential properties of something or other, where "essential" is understood as a non-modal notion along the lines of Fine (1994). A paradigm case: it is allegedly essential to \{Socrates\} that it contain Socrates, but not essential to Socrates that he belong to \{Socrates\} (while it is necessary of Socrates that he belong to \{Socrates\}). Call this the Essentialist Reply. According to the Essentialist Reply, in developing any counterfactual scenario, we must keep the distribution of essential properties fixed. Thus, because having atomic number 79 is an essential property of gold, we may import (G) into the scope of $\neg(\mathrm{G})$.

The Essentialist has some work to do in order to convince us that his solution respects Anti-exceptionalism: he must explain by what ordinary cognitive capacity it is that we are capable of discriminating between essential properties and others. Perhaps the most promising line to take here is to argue that this discriminatory capacity is in evidence in scientists' differential interest in 'deep explanatory properties" such as atomic numbers over superficial ones such as malleability. However, the extreme unclarity of the notion of essence-at present-does not inspire much confidence in this project. The investigation of the logic and metaphysics of essence is only in its infancy ${ }^{17}$; perhaps some day these will be as clear and useful as philosophical tools as the logic and metaphysics of modality are now, but until then the Essentialist Reply amounts to little more than a promissory note.

It is worth noting at this juncture that if Roca-Royes (2011) were right that the Essentialist Reply requires Williamson to attribute knowledge of essence to those who succeed in attaining knowledge of the necessity of non-identity PMNs via CD, the Essentialist Reply would be quite clearly hopeless. First, it is difficult to see how attributions of knowledge of essence are consistent with Anti-exceptionalism. Secondly, and more importantly, because essence ascriptions entail ascriptions of necessity, if a successful $C D$ of $\neg(G)$ into (G) required us to know that having

17 Fine $(1994,1995,2001)$ are the key papers. 
atomic number 79 is essential to gold, we could infer the necessity of (G) directly from this fact, and there would be no need to engage in CD.

However, Williamson can avoid attributions of knowledge of essence by stating his position as I did two paragraphs ago. Roca-Royes, for her part, makes use of the premise that "if our counterfactual judgments are to amount to counterfactual knowledge, it cannot be a matter of chance that we just happen to hold fixed (the) constitutive facts", and moreover we "must knowledgeably hold them fixed" (38, original emphases). This is an implausible requirement, as we can see by considering an analogy with perception. For your judgment that the patch of grass is green to count as knowledge, it is not sufficient that your visual system just by chance presents you with a green experience when you glance at the grass- that much is true. But it would be highly implausible to say that you do not know that the patch of grass is green unless you are knowledgeably sensitive to the right wavelengths. Something short of knowledge is enough: e.g., for a reliabilist it suffices that your visual system is reliably sensitive to the appropriate wavelengths.

The fourth option might be thought of as variant of the Essentialist Reply-call it Essentialism 2: that constitutive facts are identity and distinctness facts, of first as well as higher orders. (One fairly common gloss on the notion of essential property is that an essential property is one that pertains to the identity of something.) Thus, we can show $\mathrm{H}=\mathrm{P}$ to be necessary by making the counterfactual supposition that $\mathrm{H} \neq \mathrm{P}$, and importing the fact that $\mathrm{H}=\mathrm{P}$ into its scope. And similarly for secondorder identities, because many of the standard examples of PMNs are logical consequences of property identities, which may, qua constitutive facts, be imported into the scope of any counterfactual supposition whatever. Similarly, mutatis mutandis, for distinctness facts as well. Essentialism 2 promises to deliver via counterfactual development knowledge of the necessity of the same range of paradigm PMNs whose necessity I showed in $\S 4$ by deductive argument.

But can it do so? If Essentialism 2 is correct, then $\mathrm{H} \neq \mathrm{P}$ is cotenable with $\mathrm{H}=\mathrm{P}$, and the following is a fine counterfactual development.

(1) Suppose that Hesperus were not Phosphorus. Then Hesperus would be Phosphorus.

But (1) does not seem like a fine counterfactual development. To repeat, while it may be part of our ordinary practice of evaluating counterfactuals to generally import true identities into the scopes of counterfactual suppositions, it is no part of our ordinary practice of evaluating counterfactuals to import any proposition into the scope of a counterfactual supposition of its own negation. Some explanation as to why this departure from our ordinary practice is permissible is required in the case of "constitutive facts", identified here with identity and distinctness facts. What might it be?

One suggestion can be extrapolated from Williamson's reply to those who "insist that in imaginatively developing the counterfactual supposition that Hesperus is not Phosphorus, we are committed to the explicit denial of no logical truth, as in the consequent of (32)" (PoP, p. 174), where (32) is:

(32) If Hesperus had not been Phosphorus, then Phosphorus would not have been Phosphorus. 
Williamson says that such critics "will presumably accept this trivial instance of reflexivity":

(33) If Hesperus has not been Phosphorus, then Hesperus would not have been Phosphorus.

But we also have

(35) Hesperus = Phosphorus,

and "coreferential names are intersubstitutable in counterfactual contexts", so "the argument from (33) and (35) to (32) should be valid" (PoP, pp. 174-175, emphasis mine).

Here Williamson explains why $\mathrm{P} \neq \mathrm{P}$ can legitimately be imported into the scope of the counterfactual supposition that $\mathrm{H} \neq \mathrm{P}$ : it is because "coreferential names are intersubstitutable in counterfactual contexts" and $\mathrm{H}=\mathrm{P}$, so ' $\mathrm{H}$ ' and ' $\mathrm{P}$ ' are coreferential. $\mathrm{H} \neq \mathrm{P} \square \longrightarrow \mathrm{H} \neq \mathrm{P}$ is a logical truth, and a substitution of ' $\mathrm{H}$ ' for ' $\mathrm{P}$ ' in its consequent, which is (presumably logically) "valid", yields $\mathrm{H} \neq \mathrm{P}$ $\square \longrightarrow \mathrm{P} \neq \mathrm{P}$. The explanation, then, makes use of the applicability of Leibniz's Law in counterfactual contexts. Presumably the explanation of why it is legitimate to import $\mathrm{H}=\mathrm{P}$ into the scope of $\mathrm{H} \neq \mathrm{P}$ would make use of the same logical fact: from the empirically known $\mathrm{H}=\mathrm{P}$ and the logical truth $\mathrm{H} \neq \mathrm{P} \square \mathrm{H}=\mathrm{H}$ (or $\mathrm{H} \neq \mathrm{P} \square \longrightarrow \mathrm{P}=\mathrm{P}$ ) one can validly move to $\mathrm{H} \neq \mathrm{P} \square \longrightarrow \mathrm{H}=\mathrm{P}$. Let us suppose that this adequately explains why the importation of $\mathrm{H}=\mathrm{P}$ into the scope of $\mathrm{H} \neq \mathrm{P}$ is legitimate (in fact, I think it does). The problem is that, once we have the explanation, there is no need for such exportation, because we have already shown the counterfactual $\mathrm{H} \neq \mathrm{P} \square \mathrm{H}=\mathrm{P}$ to be true by deducing it from known premises. Counterfactual development is an idle wheel here-all of the epistemological work is being done by the deductive argument.

There is one area in which my reply to the Humean has no clear advantage over Williamson's: alleged PMNs which appear not to be reducible to identity or distinctness facts-let us call them seemingly irreducible propositions (SIPs). For example, any case of the necessity of origin or material constitution is a putative SIP: that (if George W. Bush exists, then) George H. W. Bush is the father of GWB; that Kripke's lectern is not made of ice; etc.

But does Williamson's approach have any advantage over mine when it comes to SIPs? One might worry that it does. Perhaps, one might worry, the most pressing task of contemporary modal epistemology is in fact to explain how we can know SIPs to be necessary, since the most widely discussed PMNs in the modal metaphysics literature are SIPs. ${ }^{18}$ And Williamson does have a story, however terse, about how one might arrive at knowledge of the necessity of a SIP: SIPs are constitutive facts, which may be imported into the scope of any counterfactual supposition whatever. I, it may seem, do not even have a terse story.

But in fact I do have a story-a terse one, like Williamson's—about how one might come to know a SIP to be necessary. SIPs are, after all, only seemingly irreducible: given a widely shared and plausible conception of properties, any true

\footnotetext{
${ }^{18}$ I would like to thank an anonymous referee for raising this worry.
} 
necessity claim is a logical consequence of a true property identity. For suppose, as I do, that intensional equivalence is sufficient for property identity. ${ }^{19}$ Then the truth of $(3) /(4)$ guarantees the truth of $(3=) /(4=)$ :

(3) $\square$ GHWB fathered GWB

(3=) $\lambda x(x=\mathrm{GWB})=\lambda x(x=\mathrm{GWB} \wedge$ GHWB fathered $x)$

(3) $\square \neg$ Lectern is made of ice

(4=) $\lambda x(x=$ Lectern $)=\lambda x(x=$ Lectern $\wedge \neg x$ is made of ice $)$,

and similarly for other SIPs. ${ }^{20}$ What my modal epistemology has to say about these examples is that if we know ( $3=)$, then we can come to know (3) by deducing it from (3=) via Reducibility, and similarly for (4) and (4=).

A natural objection to my account of knowledge of SIPs (one that seems to occur naturally to my interlocutors) maintains that the only way one could come to know property identities like ( $3=$ ) and $(4=)$ is by first coming to know the modal claims (3) and (4) and then deducing ( $3=)$ and $(4=)$ from them via the intensional conception of properties. The main difficulty with this claim is that it is difficult to find reasons to believe it-I know of none. I acknowledge that it seems likely that no one has come to know (3)/(4) by inferring them from $(3=) /(4=)$, but I am not aware that anyone has come to know (3)/(4) in any other way (that is, I doubt that anyone knows (3)/ (4)). What the objectors seem to have overlooked, however, is that there are a vast number of uncontentious SIPs, and it is not at all difficult to imagine coming to know many of them to be necessary by deducing them from known property identities. For example,

(5) $5>3$.

is an uncontentious SIP. While one could come to know that (5) is necessary by other means (such as the means outlined in $\S 3$ ), it would be bizarre to claim that one could not come to know (5) by deducing it via Reducibility from the known property identity

(5=) To be the successor of $3=$ to be the predecessor of 5 ,

together with the definitions of ' $>$ ' and 'predecessor'. $(5=)$ is a highly plausible claim all on its own, and to suggest that $(5=)$ could only be known by deducing it from the necessitation of (5) together with the intensional conception of properties is entirely unmotivated. Equally unmotivated is the objection that grants that we can know $(5=)$ and many other true property identities from which SIPs are deducible without doing

\footnotetext{
19 Of course no advocate of the Essentialist Reply should accept this. The Essentialist must discriminate between intensionally equivalent properties: e.g., humanity may be essential to you, but not the property of being human and Finnish or not Finnish. Since I do not advocate the Essentialist Reply, this is not a problem for me. The only other reason for adopting a hyperintensional conception of properties I am aware of is that one can believe that $\mathrm{x}$ is $\mathrm{P}$ without believing that $\mathrm{x}$ is $\mathrm{Q}$, even when $\square \forall \mathrm{x}(\mathrm{Px} \leftrightarrow \mathrm{Qx})$; however, on a structured-propositions view the components of content are more fine-grained than properties: e.g., the predicate ' $\mathrm{x}$ is $\mathrm{F}$ and $\mathrm{G}$ ' contributes to propositions both the properties $\lambda \mathrm{xFx}$ and $\lambda \mathrm{xGx}$, whereas ' $\mathrm{x}$ is $\mathrm{H}$ ' contributes only $\lambda \mathrm{xHx}$, even if $\lambda \mathrm{xHx}=\lambda \mathrm{x}(\mathrm{Fx} \wedge \mathrm{Gx})$.

${ }^{20}$ It bears noting that not only any PMN, but any true necessity claim at all, is a logical consequence of a true property identity if the standard criterion of property identity is correct. If intensionally equivalent properties are identical and $\square p$, then $\lambda x(x=x)=\lambda x(x=x \wedge p)$, which in turn entails $\square p$.
} 
any modal reasoning but maintains that, when it comes to contentious cases like (3) and (4), the relevant property identities can only be known by deducing them from the necessitations of the SIPs themselves via the intensional conception of properties. What grounds could there be for such selective skepticism about property identities? It does not help to point out that the property identities relevant to controversial putative SIPs seem difficult to know, for the necessitations of the SIPs themselves are difficult to know. And there is no advantage here for Williamson's method: it is also difficult to come to know (3) or (4) to be necessary by using CD.

And that is just as well; contentious cases are difficult to resolve-that is presumably why they are in contention. Like Williamson, I have focused on noncontentious cases-paradigmatic cases in which we take ourselves to have knowledge of necessity, but in which a skeptic denies that we have knowledgebecause that is sufficient for satisfying the desideratum of Conservatism. It is no part of the anti-skeptical project in which Williamson and I are engaged to resolve ongoing modal disputes or to offer guidance to those engaged in the disputes, or even to provide assurances that they can be resolved:

For all that has been argued here, we may in many cases be incapable of coming to know whether a given hypothesis is metaphysically possible. Philosophical controversy will naturally make these unclear cases salient (PoP, pp. 164-165).

But this "should not blind us to the wide range of clear cases" (ibid.). While some ongoing modal controversies may be irresolvable, there is no reason to believe that all of them are. Let us not forget that many of the cases that are now considered clear-that it is necessary that Hesperus is Phosphorus, that gold has atomic number 79 , and the other PMNs discussed in $\$ 4$-were once controversial. So, in fact, the Reducibility-based reply to the Humean does directly address (once) controversial cases and show that they can be resolved. The fact that the cutting edge of modal controversy has moved onward considerably in the last four decades is a reason for at least a guarded optimism about the resolvability of current modal disputes.

\section{Conclusion}

I have argued that Williamson is right that modal skeptics who are not skeptics about counterfactuals and other not specifically metaphysical subject matter, and who accept Reducibility, are committed to an incoherent position. This is an important finding, because no modal skeptic is in a position to reject Reducibility insofar as Reducibility is what we use to explain what necessity is, and modal skeptics who extend their skepticism to counterfactual and other not specifically metaphysical subject matter are embracing a much broader skepticism which should not particularly worry those engaged in modal metaphysics. However, I have argued that a reply to such modal skeptics need not make any use of the non-deductive mode of inference $(C D)$ by which we ordinarily arrive at counterfactual knowledge: for two common varieties of modal skeptic - the Quinean and the Humean-replies which produce modal knowledge by deduction from known premises are available. 
CD-based and deduction-based replies to the Quinean are both available; however, the deduction-based reply is more general and decisive. It is unclear what a CDbased reply to the Humean would be, whereas there is a straightforward and adequate deduction-based reply to the Humean, which is that the necessity of many properly metaphysical necessities deductively follows from known identity and distinctness claims. I have little informative to say about knowledge of necessity claims which are not (obvious) deductive consequences of identity or distinctness claims, but neither does Williamson.

Acknowledgments This paper is a descendant of a paper coauthored with Margot Strohminger, which we presented at various fora in 2009-2010. Even after it became a single-author project, I continued to benefit from enlightening discussions with Margot in writing the paper. My greatest debt of gratitude is to her. In addition, I owe thanks to John Hawthorne and Cian Dorr for helpful comments on earlier drafts, and to Tim Williamson, Sonia Roca-Royes, and audiences (of the coauthored ancestor paper) at the Arché Philosophical Research Centre at the University of St. Andrews, Trinity College Dublin, and the University of Oxford for helpful discussions.

\section{References}

Cresswell, M. (1990). Entities and indices. Dordrecht: Kluwer.

Fine, K. (1994). Essence and modality. Philosophical Perspectives, 8, 1-16.

Fine, K. (1995). The logic of essence. Journal of Philosophical Logic, 29, 295-313.

Fine, K. (2001). Semantics for the logic of essence. Journal of Philosophical Logic, 29, 543-584.

Fine, K. (2007). Semantic relationism. Oxford: Blackwell.

Fitch, G. W. (1976). Are there necessary a posteriori truths? Philosophical Studies, 30, 243-247.

Gendler, T. S., \& Hawthorne, J. (2002). Conceivability and possibility. Oxford: Oxford University Press.

Hill, C. S. (2006). Modality, modal epistemology, and the metaphysics of consciousness. In S. Nichols (Ed.), The architecture of the imagination. Oxford: Oxford University Press.

Jenkins, C. S. (2008). Modal knowledge, counterfactual knowledge and the role of experience. Philosophical Quarterly, 58, 693-701.

Kripke, S. (1971/1977). Identity and necessity. In S. P. Schwartz (Ed.), Naming, necessity, and natural kinds. Ithaca: Cornell University Press.

Kripke, S. (1980). Naming and necessity (2nd ed.). Oxford: Blackwell.

Lewis, D. (1973). Counterfactuals. Oxford: Blackwell.

Malmgren, A.-S. (forthcoming). Rationalism and the content of intuitive judgments. Mind.

Parsons, T. (1979). An analysis of mass terms and amount terms. In F. J. Pelletier (Ed.), Mass terms. Dordrecht: Reidel.

Predelli, S. (1998). I am not here now. Analysis, 58, 107-115.

Putnam, H. (1954). Synonymy and the analysis of belief sentences. Analysis, 14, 114-122.

Putnam, H. (1973). Meaning and reference. Journal of Philosophy, 70, 699-711.

Putnam, H. (1975). The meaning of 'meaning'. In Philosophical papers II: Mind, language, and reality (pp. 215-271). Cambridge: Cambridge University Press.

Roca-Royes, S. (2011). Conceivability and De Re modal knowledge. Nô̂s, 45, 22-49.

Saarinen, E. (1977). Propositional attitudes, anaphora, and backwards-looking operators. Reports from the Department of Philosophy, University of Helsinki (Vol. 6).

Segal, G. (2000). A slim book about narrow content. Cambridge: MIT Press.

Soames, S. (2002). Beyond rigidity. Oxford: Oxford University Press.

Stalnaker, R. C. (1968/1981). A theory of conditionals. In W. Harper, et al. (Eds.), Ifs: Conditionals, belief, decision, chance, and time. Dordrecht: Reidel.

Swinburne, R. (1991). Necessary a posteriori truth. American Philosophical Quarterly, 28, 113-123.

Williamson, T. (2007). The philosophy of philosophy. Oxford: Blackwell.

Yablo, S. (1993). Is conceivability a guide to possibility? Philosophy and Phenomenological Research, $53,1-42$. 